Tropical Journal of Pharmaceutical Research, December 2008; 7 (4): 1117-1121

(C) Pharmacotherapy Group, Faculty of Pharmacy, University of Benin,

Benin City, 300001 Nigeria.

All rights reserved.

Research Article

Available online at http://www.tjpr.org

\title{
Serum Complement C3 and C4 Levels in Relation to Diagnosis of Lupus Nephritis
}

\author{
N Hussain ${ }^{1 *}$, G Jaffery ${ }^{2}$, S Hasnain \\ ${ }^{1}$ Department of Microbiology and Molecular Genetics, Quaid-e-Azam Campus, University of the Punjab, Lahore- \\ Pakistan, ${ }^{2}$ Department of Pathology, Services Institute of Medical Sciences, Lahore-Pakistan.
}

\begin{abstract}
Purpose: The main objective of this study was to measure serum complement C3 and C4 concentrations in patients of lupus nephritis to see if these simple measurements would give useful information to the clinician managing such patients..

Method: A total of 52 samples were obtained from SLE patients, 17 suffering from lupus nephritis. All patients met the revised 1997 American College of Rheumatology criteria for SLE. Serum C3 and C4 concentrations were measured with single gel radioimmunodiffusion technique.

Results: In lupus nephritis, C3 and C4 are generally correlated. Both C3 and C4 levels were decreased but $\mathrm{C} 4$ concentrations were more often and more profoundly depressed than C3 concentration.

Conclusion: All patients of lupus nephritis with low C3 or C4 concentrations should have serial measurements performed and selected patients will need a full complement profile, including measurement of alternate pathway components and total hemolytic pathway.
\end{abstract}

Key Words: Systemic lupus erythematosus, Auto antibodies, Lupus nephritis, C3 and C4

Received: 18 June 2008

Revised accepted: 25 September 2008

${ }^{*}$ Corresponding author: Department of Microbiology and Molecular Genetics, Quaid-e-Azam Campus, University of the Punjab, Lahore-Pakistan. 


\section{INTRODUCTION}

Systemic Lupus Erythematosus (SLE) is a relatively common autoimmune disorder that results in the production of an array of autoantibodies ${ }^{1,2}$. The complement system consists of 11 components which together form some $10 \%$ of circulating plasma protein. The association between the complement system and SLE is contradictory ${ }^{3,4}$. The complement system has long been known to be activated in exacerbations of SLE, particularly reflecting nephritic activity ${ }^{5,6,7}$. It has been debated whether this complement activation is important in the pathogenesis of SLE or whether it is an innocent epiphenomenon. Complement is a mediator of inflammation, complement deficiency predisposes to the development of SLE. Inherited complement C4 deficiency, whether partial or complete, confer a high risk to developing SLE, whereas C3 deficiency is only rarely associated with SLE-like illness ${ }^{8,9}$.

The association between complement deficiencies and SLE supports an important role of complement in preventing immune complex mediated tissue damage ${ }^{10}$. However, increased activation of complement without deficiencies may also contribute to the tissue damage in SLE, since it was recently shown in an experimental mouse SLE model that specific inhibition of complement abolished the development of the disease ${ }^{11,12}$.

The role of complement in lupus was examined in mouse models. The first model is based on the hypothesis that complement plays a substantial role in the clearance of immune complexes and/or apoptotic debris, which is thought to be impaired in lupus. Apoptotic bodies are a potential major source of lupus self antigens, and failure to remove them could lead to inappropriate production of pathogenic autoantibodies. Support for a role for early complement in clearance of apoptotic debris comes from the finding that mice deficient in $\mathrm{C} 4$ have defects in the clearance of injected apoptotic thymocytes. Moreover mice deficient in C4 spontaneously develop autoantibodies against nuclear antigens. The presence of increased apoptotic bodies in the affected glomeruli of C4 deficient mice supports this model ${ }^{13,14}$.

A second nonexclusive model proposes that the innate immune system, including complement, is protective against lupus by enhancing negative selection of self-reactive $B$ cells. According to this hypothesis, innate proteins enhance the localization of lupus antigens such as dsDNA and nuclear proteins within the primary lymphoid compartment. Efficient presentation of self-antigens results in elimination or editing of potential selfreactive $\mathrm{B}$-cells. Thus deficiency in innate proteins such as serum amyloid protein, DNAse I, natural IgM, C1q or C4 could lead to an escape from negative selection of selfreactive $B$ cells and their potential activation in the periphery in the presence of cognate $\mathrm{T}$ cell help and lupus antigen. ${ }^{15,16}$

We have measured C3 and C4 concentrations using commercially available immunodiffusion plates to see if these readily available, simple measurements would give useful information to the clinician managing patients with lupus nephritis.

\section{MATERIALS AND METHODS}

Altogether fifty-two blood samples were obtained from SLE patients during the period February 2007 - February 2008. All the patients included in this prospective study fulfilled 4 or more of the American Rheumatism Association Classification Criteria for SLE. Of the 52 SLE patients, 17 were cases of lupus nephritis. Blood samples were obtained in an empty vacutainer tubes for the preparation of serum. Serum was obtained by allowing the blood to clot at room temperature for two hours and the tube was then centrifuged. Serum was removed, aliquoted and stored at $-70^{\circ} \mathrm{C}$. The $\mathrm{C} 3$ and $\mathrm{C} 4$ concentrations were usually obtained within one week of receipt. Consent was taken from all patients before blood sampling. This research was approved by the ethical committee of Punjab University, LahorePakistan.

C3 and C4 were measured by using immunodiffusion plates (FAR Ven Fermi, 12Italy) as described by the manufacturer. 
These tests define serum proteins by single radial immunodiffusion plate. An amount of serum was placed in the agarose well, containing a mono-specific antibody. The serum spreads in the agarose and the contained antigen forms an immune complex with the antibody. A precipitation ring whose dimension is proportional to the concentration of protein in the serum was formed. Sample precipitation diameter was measured and the reference value table was used to read the concentration of the protein ${ }^{17}$. Other immunological techniques for the confirmation of diagnosis of SLE (ANA, dsDNA, Sm, SSA, SSB, anti-Histone, RibP) were preformed by indirect ELISA technique (Orgentec ELISA kits).

\section{RESULTS}

A total of 52 patients with SLE were enrolled in our study. Of the 52, 17 were cases of lupus nephritis. Patients were diagnosed as having SLE based on the 1982 revised criteria proposed by the American College of Rheumatology ${ }^{18}$. In our lupus nephritis patients, fourteen (82.35\%) were females and three $(17.64 \%)$ were males.

Mean age at entry to the study was $28.1 \mathrm{yrs}$ (range 11-60). C3 and C4 were measured by using immunodiffusion plates. Serum C3 and C4 concentrations (C3 $=50-120 \mathrm{mg} / \mathrm{dl}, \mathrm{C} 4=$ $20-50 \mathrm{mg} / \mathrm{dl})$ were normal in four $(23.5 \%)$ lupus nephritis patients. Low $\mathrm{C} 3$ and normal C4 concentrations were found in only two patients (11.7\%). Normal C3 and low C4 concentrations $(35.29 \%)$ were commonest in lupus nephritis group as shown in Fig 1. Five patients $(29.4 \%)$ with lupus nephritis showed low C3 in the presence of low C4 concentration. These findings are summarized in Fig 2.

The results of the laboratory investigations are shown in Table 1. Renal disorder was characterized by proteinuria and red cell cast. Hematological disorders include normocytic normochromic anaemia (64.7\%), elevated ESR (100\%), thrombocytopenia (23.52\%) while total Leukocyte count, differential leukocyte count were normal. Immunological parameters like ANA, dsDNA were performed by an indirect ELISA technique; positive ANA was observed in $88.23 \%$ while positive dsDNA was found in $82.35 \%$ of the lupus nephritis patients. High urea (70.58\%) and high creatinine $(88.23 \%)$ levels were the best indicators suggestive of lupus nephritis. Lupus nephritis patients were treated according to the disease activity.

\section{DISCUSSION}

Of the components of the "classical pathway" of complement activation, the easiest to measure is $\mathrm{C} 4$. Its measurement together with that of C3 should enhance the understanding of the mechanisms involved and aid the clinical definition of lupus nephritis. C3 is a good screening test for lupus patients with renal disease.

Lupus nephritis is generally considered to be the best established human model of chronic soluble complex renal disease ${ }^{19}$. It is not surprising; therefore to find that $\mathrm{C} 4$ was depleted more than $\mathrm{C} 3$, since soluble complexes activate the classical pathway.

C4 concentration was low in most of the lupus nephritis patients, but this was not associated with increased complement activation. Low C4 levels may falsely be regarded as classical pathway activation. Several other factors may explain low C4 values in SLE. Partial defects or homozygous defects in either C4A or/and C4B will result in reduced levels of total C4. Reduced synthesis or increased catabolism of C4 without corresponding complement activation may also explain low C4 values.

In 2002, Eniav found that the development of severe SLE in the absence of both classical and alternative complement pathways suggests that it is the absence of $\mathrm{C} 4$, and not the presence of C3 that is critical in SLE pathogenesis $^{20}$. Thus, this study supports our research in the reference that complement $\mathrm{C} 4$ provides an important protective role against the development of SLE. Cameron studied 55 samples of Lupus nephritis and he also found that low plasma C4 concentrations were commonest in lupus nephritis group, but a C3 concentration of below $20 \%$ of reference normal serum was not seen in their study ${ }^{21}$. In 1999, Mollnes found that the complement 


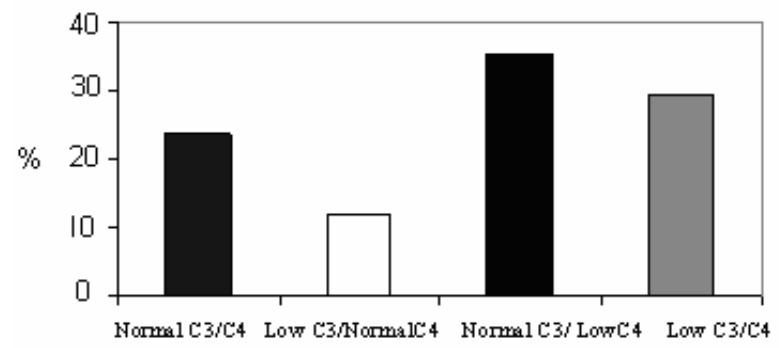

Fig 1: Proportion of $\mathrm{C} 3 / \mathrm{C} 4$ in lupus nephritis patients

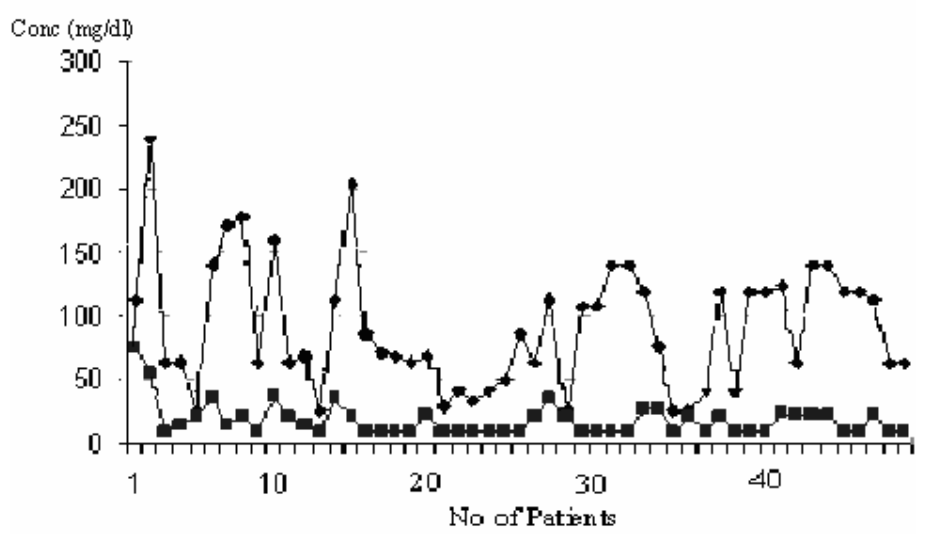

Fig 2: Levels of C3 and C4 in lupus patients. C3: • C4: •

Table 1: Hematological, biochemical and immunological parameters in lupus nephritis patients

\begin{tabular}{ll}
\hline PARAMETERS & PERCENTAGE (\%) \\
\hline Hematological & $100 \%$ \\
ESR & $64.7 \%$ \\
Anemia & $23.52 \%$ \\
Thrombocytopenia & \\
Biochemical: & $70.58 \%$ \\
High Urea & $88.23 \%$ \\
High Creatinine & \\
Immunological & $88.23 \%$ \\
Positive ANA & $82.35 \%$ \\
Positive dsDNA & \\
\hline
\end{tabular}

tests are of limited importance in routine examination of SLE without nephritis, and terminal complement complex was suggested to be one of the most sensitive markers for disease activity ${ }^{22}$. This study tells us the importance of measuring C3 and C4 concentrations in lupus nephritis patients because $\mathrm{C} 3$ and $\mathrm{C} 4$ reflect the level of the circulating components irrespective of their functional state. 
From this data, it is suggested that all patients with lupus nephritis should have the C3 concentration of their plasma or serum estimated. All those with a low C3 or suspected lupus nephritis should have C4 estimation in addition. All patients with low C3 or C4 concentrations should have serial measurements performed to see if the alterations reverse within a few weeks or persist.

Most patients with a persistently low C3 or C4 concentration will need a renal biopsy at some point and should of course be measured for lupus nephritis. Selected patients will need a full complement profile, including measurement of alternate pathway components and total hemolytic pathway.

\section{ACKNOWLEDGEMENT}

We would like to thank rheumatologists, physicians and pediatricians who referred lupus patients to us or kindly supplied samples obtained from their patients.

\section{REFERENCES}

1. Boumpas DT. Systemic Lupus Erythematosus: emerging concepts. Ann Intern Med 1995:122:940.

2. Mills JA. Systemic Lupus Erythematosus. New Eng J Med 1994;330:1871-1872.

3. Kotzin BL. Systemic Lupus Erythematosus. Cell Biology 1986;85:303-306.

4. McShane DJ, Rothfield NJ. The 1982 revised criteria for the classification of SLE. Rheum Dis 1986; 45:435-437.

5. Walport MJ, Davies KA. Complement and immune complexes. Res Immunol 1996; 147:103.

6. Morgan BP, Walport MJ. Complement deficiency and disease. Immunol Today 1991;12:301.

7. Rabbani MA, Tahir MH, Siddiqui BK, Ahmad B, Shamim $A$, Shah MA, Ahmad A. Renal involvement in systemic lupus erythematosus in Pakistan. J Pak Med Assoc 2005;55:328-32.

8. Fielder $A H$, Walport MJ, Batchelor JR, Rynes RI, Balck CM, Dodo IA, Hughes GR. Family study of the major histocompatibility complex in patients with SLE: importance of null alleles of $C 4 A$ and $C 4 B$ in determining disease susceptibility. Br J Med 1983;286:425.

9. Walport MJ, Davies KA, Morley BJ, Botto $B$.
Complement deficiency and autoimmunity. Ann NY Acad Sci 1997; 815:267.

10. Chen Z, Karolov SB, Kelsoe G. Complement C4 inhibit systemic autoimmunity through a mechanism independent of complement receptors CR1 and CR2. J Exp Med 2000;192:1339.

11. Prodeus AP, Goerg S, M Shen O, Pozdnyakova O, Chu E, Alicot EM, Goodnow CC, Carroll MC. A critical role for complement in maintainence of self-tolerance. Immunity 1998;9:721.

12. Theofilopoulos AN, Dixon FJ. Murine models of systemic lupus erythematosus. Adv Immunol 1985;37:269.

13. Sekine H, Reilly CM, Molano ID, Garnier G, Circolo $P$, Ruiz VM, Holers S, Boackle A, Gilkeson GS. Complement component $\mathrm{C} 3$ is not required for full expression of immune complex glomerulonephritis in MRL/pr mice. J Immunol 2001;166:6444.

14. Atkinson JP. Complement activation and complement receptors in systemic lupus erythematosus. [Review] Springer Semin Immunopathol 1986;9:179-94.

15. Davies KA, Peters AM, Beyon $H L$, Walport MJ. Immune complex processing in patients with SLE. In vivo imaging and clearance studies. J Clin Invest 1992;90:2075-2083.

16. Porcel JM, Ordi J, Castrosalomo A, Vilardell $M$, Rodrigo MJ, Gene T. The value of complement activation products in the assessment of systemic lupus erythematosus flares. Clin Immunol Immunopathol 1995;74:283-8.

17. Cohen M. Systemic Lupus Erythematosus: diagnosis and classification. Intern Med J 2004;34(12):701-2.

18. McShane DJ, Rothfield NJ. The 1982 revised criteria for the classification of SLE. Rheum Dis 1986;45:435-437.

19. Auda G, Holme ER, Davidson JE, Zoma A, Veitch J, Whaley K. Measurement of complement activation products in patients with chronic rheumatic diseases. Rheumatol Int 1990;10:185-9.

20. Eniav S, Pozdnyakova O, Ma M, Carroll MC. Complement C4 is protective for Lupus Disease Independent of C3. Am Assoc Immunol 2002;168:1036-1941.

21. Cameron JS, Vick RM, Ogg CS, Seymour WM, Chantler C, Turner DR. Plasma C3 and C4 Concentrations in Management of Glomerulonephritis. Br Med J 1973;3:668-672.

22. Mollnes TE, Haga HJ, Brun JG, Nielsen EW, Sjoholm A, Sturfeldt G, Martensson U, Bergh K, Rekvig OP. Complement activation in patients with systemic lupus erythematosus without nephritis. Br Soc Rheumatol 1999;38:933-940. 